International Journal of Pure and Applied Mathematics

Volume 84 No. 5 2013, 497-516

ISSN: 1311-8080 (printed version); ISSN: 1314-3395 (on-line version)

url: http://www.ijpam.eu

doi: http://dx.doi.org/10.12732/ijpam.v84i5.4

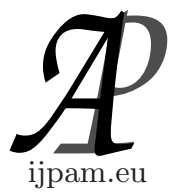

\title{
FINITE SOLVABLE GROUPS HAVING A UNIQUE IRREDUCIBLE CHARACTER OF A GIVEN DEGREE
}

\author{
Venkata Rao Potluri \\ Department of Mathematics \\ Reed College \\ 3203, SE Woodstock Blvd, Portland, OR 97202, Portland, USA
}

\begin{abstract}
It has been conjectured that $\operatorname{PSL}(2, q)$, the projective special linear group of $2 \times 2$ matrices over a field of order $q$, is the only non-solvable group satisfying the property that it has a unique irreducible complex character $\chi$ of degree $m>1$ and every other irreducible complex character is such that its degree is relatively prime to $m$. (Such a $\chi$ is a particular case of the Steinberg character of finite Chevalley groups.) In this paper, we consider finite solvable groups satisfying the above property and obtain a complete classification.
\end{abstract}

AMS Subject Classification: 20C15

Key Words: finite solvable groups, Chevalley groups, Steinberg character, irreducible complex characters

\section{Introduction}

If $G$ is a finite simple Chevalley group, then $G$ has an irreducible complex character $\chi$ whose degree is the order of a $p$-Sylow subgroup of $G$, and all other irreducible complex characters are in the principal $p$-block. Such a $\chi$ is called the Steinberg character. See [1] and [3]. For simple groups $G$, this property seems to hold only if $G$ is a finite simple Chevalley group. The conjecture, then, is that the above property about the irreducible complex characters of $G$

Received: October 10, 2012

(c) 2013 Academic Publications, Ltd. url: www.acadpubl.eu 
forces $G$ to be a finite simple Chevalley group. This conjecture seems to be too difficult at present. However, $\operatorname{PSL}(2, q)$ is the only known non-solvable group which satisfies the following stronger property:

Property 1.1. There is a unique irreducible complex character $\chi$ of degree $m>1$ and every other irreducible complex character is such that its degree is relatively prime to $m$.

So the problem of classifying all finite groups $G$ satisfying Property 1.1 can be viewed, at least in the simple case, as a first step towards classifying finite simple Chevalley groups by their Steinberg character.

A natural and more general question that arises is "What are all the finite groups that satisfy Property 1.1?" In this paper we obtain a complete classification of all finite solvable groups satisfying Property 1.1 (see Theorem $3.1)$.

\section{Notations and Preliminary Results}

In this section we set the needed notations and state without proof some well known results in character theory which are needed in the proof of the main theorem. A reference is given in each case. All groups considered in this paper are finite. For a group $G$ :

(i) $Z(G)$ is the center of $G$.

(ii) $G^{\prime}$ is the derived group of $G$.

(iii) $[G: H]$ is the index of a subgroup $H$ in $G .|H|$ and $|x|$ are orders of a subgroup $H$ and an element $x$ in $G$, respectively.

(iv) If $H \leq G$ and $x, y \in G$, then $H^{x}=x^{-1} H x$ and $y^{x}=x^{-1} y x$.

(v) If $\psi$ is a character of a subgroup $H$, and $x \in G$, then $\psi^{x}$, defined on $H^{x}$ by $\psi^{x}\left(h^{x}\right)=\psi(h), h \in H$, is a character of $H^{x}$. If $H$ is normal in $G$, the stabilizer of $\psi=\left\{x \in G \mid \psi^{x}=\psi\right\}$ is denoted by $T_{\psi, H}$ or simply by $T_{\psi}$ if the domain of $\psi$ is clear from the context.

(vi) $\psi^{G}$ is the character of $G$ induced from the character $\psi$ of the subgroup $H .\left.\chi\right|_{H}$ is the restriction to $H$ of a character $\chi$ of $G$.

(vii) $\operatorname{ker} \chi$ is the kernel of a representation corresponding to the character of $G$. 
(viii) The inner product of two class functions $\alpha$ and $\beta$ on $G$ is defined by $(\alpha, \beta)_{G}=|G|^{-1} \sum_{g \in G} \alpha(g) \overline{\beta(g)}$. In particular, if $\alpha$ is a character and $\beta$ an irreducible character of $G$, then $(\alpha, \beta)_{G}$ is the multiplicity of $\beta$ in $\alpha$.

We assume all characters to be complex characters. Throughout, $C_{n}$ denotes the cyclic group of order $n$.

We now present the needed results without proofs. A reference is provided in each case.

Lemma 2.1 (Frobenius reciprocity law, [5] Theorem 9.4). If $H$ is a subgroup of $G$ and $\theta$ and $\eta$ are complex valued class functions of $H$ and $G$ respectively, then $\left(\eta, \theta^{G}\right)_{G}=\left(\left.\eta\right|_{H}, \theta\right)_{H}$.

Lemma 2.2 ([5] Theorem 9.10). Let $H$ be a normal subgroup of $G$. If $\eta$ is an irreducible character of $G$, then there exists an irreducible character $\theta$ of $H$ and a positive integer $a$ such that $\left.\eta\right|_{H}=a \sum_{i=1}^{t} \theta^{g_{i}}$ where $t=\left[G: T_{\theta}\right]$ and $\left\{g_{i}\right\}$ is a complete system of coset representatives of $T_{\theta}$ in $G$.

Lemma 2.3 ([2] Corollary 45.5). Let $\theta$ be an irreducible character of a normal subgroup $H$ of $G$. Then $\theta^{G}$ is irreducible if and only if $T_{\theta}=H$.

Lemma 2.4 ([12] Theorem 1.9). Suppose $G$ is a solvable group, $p$ a prime such that $(p, \psi(1))=1$ for each irreducible character $\psi$ of $G$. Then $G$ has a normal abelian $p$-Sylow subgroup.

Lemma 2.5 (Thompson [13] Theorem 1). Suppose $G$ is any finite group, $p$ a prime such that $p \mid \psi(1)$ for each non-linear irreducible character $\psi$ of $G$. Then $G$ has a normal p-complement.

Lemma 2.6 ([5] Theorem 9.13). If $N$ is a normal and abelian subgroup of $G$ and $\psi$ is any irreducible character of $G$, then $\psi(1) \mid[G: N]$.

Lemma 2.7 ([5] Theorem 12.1). Let $A=\left(a_{i j}\right)$ be a nonsingular complex matrix of degree $k$. If $\sigma$ is a permutation of the $k^{2}$ ordered pairs $(i, j)$ with $i, j=1,2, \ldots, k$, define $A^{\sigma}=\left(a_{\sigma(i, j)}\right)$. Suppose $G$ is a group of permutations on the $k^{2}$ ordered pairs $(i, j)$, such that for each $\sigma$ in $G, A^{\sigma}$ can be obtained from $A$ by permuting the rows of $A$ and $A^{\sigma}$ can also be obtained from $A$ by permuting the columns of $A$. Let $C$ be the columns of $A$ and $R$ the rows of $A$. Then $G$ acts on $C$ and $R$ as a permutation group, and the number of orbits is the same in both representations. Moreover, if $G$ is cyclic, then the number of rows fixed by $G$ equals the number of columns fixed by $G$.

In all applications of the above lemma in this paper, $A$ will be the character table of a normal subgroup. 
Lemma 2.8 ([12] Proposition 1.5). Let $G$ be a group and $N \triangleleft G$. Then $G$ is Frobenius with kernel $N$ if and only if each non-principle irreducible character of $N$ induces an irreducible character of $G$.

Lemma 2.9 (Gallagher [6] Theorem 2). Let $N \triangleleft G, \tau$ be an irreducible character of $N$. Suppose $\tau$ has an extension $\hat{\tau}$ to $T_{\tau}$. Then $\tau^{G}=\sum_{\omega} \omega(1)(\omega \hat{\tau})^{G}$ where the sum runs over the irreducible characters $\omega$ of $T_{\tau} / N$. Each $(\omega \hat{\tau})^{G}$ is irreducible and $\left(\omega_{1} \hat{\tau}\right)^{G}=\left(\omega_{2} \hat{\tau}\right)^{G}$ implies $\omega_{1}=\omega_{2}$.

Lemma 2.10 (Gallagher [6] Theorem 1). Let $N \triangleleft G$ and $\tau$ be an irreducible character of $N$. Then for each irreducible constituent $\chi$ of $\tau^{G}$, there is a unique irreducible character $\zeta$ of $T_{\tau}$ such that $\left(\tau^{T_{\tau}}, \zeta\right)_{T_{\tau}} \geq 1$ and $\left(\zeta^{G}, \chi\right)_{G} \geq 1$. For this $\zeta,\left.\zeta\right|_{N}=a \tau$ for some positive integer $a$ and $\zeta^{G}=\chi$.

Lemma 2.11 (Gallagher [6] Theorem 3). Let $N$ be a normal subgroup of prime index $p$ in $G$. Then each invariant irreducible character of $N$ extends to an irreducible character of $G$.

Lemma 2.12 (Gallagher [6] Lemma 1 and Theorem 6). Suppose $N \unlhd G$. If $N$ is a Hall subgroup of $G$, then each irreducible character of $N$ extends to its stabilizer. If $N$ is abelian and complemented in $G$, then each irreducible character of $N$ extends to its stabilizer.

Lemma 2.13 (Isaacs [10] Proposition 3). Let $K \triangleleft H$ be such that $H / K$ is an elementary abelian p-group and let $A$ act on $H$ and normalize $K$, with the induced action on $H / K$ being non-trivial and irreducible. Then the following hold.

(i) If $\chi \neq 1_{H}$ is an irreducible character of $H$ which is stabilized by $A$, we have that either:

(a) $\left.\chi\right|_{K}$ is irreducible and $\neq 1_{K}$, or

(b) $\left.\chi\right|_{K}=a \theta, \theta$ irreducible, $\neq 1_{K}$ and $a^{2}=[H: K]$, or

(c) $\left.\chi\right|_{K}=\sum_{i=1}^{t} \theta_{i}$ where $t=[H: K]$ and the $\theta_{i}$ are distinct irreducible characters of $K$, transitively permuted by $H$.

(ii) If $\theta \neq 1_{K}$ is an irreducible character of $K$ which is stabilized by $A$ and $\theta^{H}$ is the induced character, we have that either:

(a) $\theta^{H}$ is irreducible, or

(b) $\theta^{H}=a \chi, \chi$ irreducible and $a^{2}=[H: K]$, or

(c) $\theta^{H}=\sum_{i=1}^{t} \chi_{i}$ where $t=[H: K]$ and the $\chi_{i}$ are distinct irreducible characters of $H$ which are transitively permuted by multiplication by the linear characters of $H / K$. 
In all applications of the above lemma in this paper, we let $H / K$ be a chief factor of a solvable group $G$ and let $A=G$. We also note from the proof that the assumption that the action of $A$ is non-trivial on $H / K$ is used only to show $\theta \neq 1$ in (b) of (i).

Lemma 2.14 (Seitz [11]). A group $G$ has exactly one non-linear, irreducible character if and only if (i) $G$ is an extra-special 2-group or (ii) $G$ is isomorphic to the group of all transformations $x \mapsto \alpha x+\beta, \alpha \neq 0$ on a field of order $p^{n} \neq 2$.

The following lemma is needed in the proof of the next lemma, which will play an important role in the proof of the main theorem in the next chapter.

Lemma 2.15. Let $G=H A$ be a Frobenius group with kernel $H$ and complement $A$. Suppose $A$ is cyclic. Let $V$ be a faithful, irreducible module of $\mathbb{C} G$, the group algebra over complex numbers. Then $\left.V\right|_{A}=t \cdot \rho_{A}$ where $\rho_{A}$ is the regular representation of $A$ and $t$ is some positive integer.

Proof. By Lemma 2.2, $\left.V\right|_{Z(H)}=V_{1}+V_{2}+\cdots+V_{\ell}$ where the $V_{i}$ are homogeneous and transitively permuted by $A$.

Claim. No non-identity element of $A$ stabilizes $V_{i}$ for any $i$.

Suppose that the subgroup $A_{1}$, the stabilizer of $V_{1}$ in $A$, is non-trivial. Then $G_{1}=Z(H) A_{1}$ is a Frobenius group. Let $N_{1}$ be the kernel of the representation of $G_{1}$ on $V_{1}$. Since $G_{1}$ is Frobenius, either $N_{1}<Z(H)$ or $N_{1} \geq Z(H)$. If $N_{1} \geq Z(H)$, then, sine the $V_{i}$ 's are transitively permuted by $A, Z(H)$ is contained in the kernel of the representation of $Z(H)$ on every $V_{i}$, contradicting the hypothesis that $V$ is a faithful module for $G$. So $N_{1}<Z(H)$ and $G_{1} / N_{1}=Z(H) / N_{1} \cdot A_{1} N_{1} / N_{1}$ is a Frobenius group. Since $G_{1} / N_{1}$ acts faithfully on $V_{1}, Z(H) / N_{1}$ is cyclic and each element of $Z(H) / N_{1}$ acts as a scalar transformation on $V_{1}$. Therefore $\left[Z(H) / N_{1}, A_{1} N_{1} / N_{1}\right]$ is in the kernel of the representation of $G_{1} / N_{1}$ on $V_{1}$, and hence, $\left[Z(H) / N_{1}, A_{1} N_{1} / N_{1}\right]=1$. This is a contradiction since $G_{1}$ is Frobenius. This proves the claim.

If $v \neq 0 \in V_{1}$, the elements in the set $\left\{v^{a} \mid a \in A\right\}$ lie in distinct direct summands $V_{i}$ by our claim and, hence, are linearly independent. Since $\left(v^{a}\right)^{a^{\prime}}=v^{a a^{\prime}}$, the space spanned by $\left\{v^{a} \mid a \in A\right\}$ gives a regular representation of $A$. Now let $\left\{v_{1}, v_{2}, \ldots, v_{t}\right\}$ be a basis of $V_{1}$. Then $\left\{v_{i}^{a} \mid 1 \leq i \leq t, a \in A\right\}$ is a basis of $V$ and

$$
\left.V\right|_{A}=\left\langle\left\{v_{1}^{a} \mid a \in A\right\}\right\rangle \oplus\left\langle\left\{v_{2}^{a} \mid a \in A\right\}\right\rangle \oplus \cdots \oplus\left\langle\left\{v_{t}^{a} \mid a \in A\right\}\right\rangle
$$

is a direct sum of $t$ submodules, each giving a regular representation of $A$. Thus, $\left.\theta\right|_{A}=t \cdot \rho_{A}$. 
Lemma 2.16. Let $K$ be minimal normal in a solvable group $G$ and let $G / K$ be Frobenius with kernel $M / K$. Suppose that $G / M$ is cyclic of order $q-1$ where $q=p^{n}$ for some prime $p$ and $M / K$ is elementary abelian of order $q$ but $M$ is not abelian. Then one of the following assertions holds:

(a) $q=3$

(b) $K$ is a $p$-group (so that $M$ is a $p$-group)

(c) There exists an irreducible character $\varphi \neq 1_{K}$ of $K$ and an irreducible character $\theta$ of $G$ such that $\left(\varphi^{G}, \theta\right)_{G} \geq 1$ and $(\theta(1), q-1) \neq 1$.

Proof. Suppose that neither (b) nor (c) holds. We shall prove that $q=3$. Let $|K|=r^{m}, r$ a prime $\neq p$, and let $Q$ be a $p$-Sylow subgroup of $G$. Then $Q \leq M$ and $M=Q \cdot K$. By the Frattini argument, $G=N_{G}(Q) \cdot M=$ $N_{G}(Q) \cdot Q \cdot K=N_{G}(Q) \cdot K$. Noting that $N_{G}(Q) \cap K \triangleleft G, K$ is minimal normal in $G$ and that $M$ is non-abelian, we get $N_{G}(Q) \cap K=1$. So $G$ is a semidirect product of $N_{G}(Q)$ and $K$ and $N_{G}(Q)$ can be written as $Q \cdot T$ where $T$ is a subgroup of order $q-1$ and is cyclic.

Let $\varphi=\neq 1_{K}$ be a linear character of $K$. By Lemma $2.12 \varphi$ extends to a linear character of $\hat{\varphi}$ of the stabilizer $T_{\varphi}$ of $\varphi$. Then $\hat{\varphi}^{G}$ is irreducible ([2] Corollary 45.4) of degree $\hat{\varphi}^{G}(1)=\hat{\varphi}(1)\left[G: T_{\varphi}\right]=\left[G: T_{\varphi}\right]$. Then $1=$ $\left(\hat{\varphi}^{G}, \hat{\varphi}^{G}\right)_{G}=\left(\hat{\varphi}^{G}, \hat{\varphi}\right)_{T_{\varphi}}$ by Lemma 2.1 and this implies $\left(\left.\hat{\varphi}^{G}\right|_{K},\left.\hat{\varphi}\right|_{K}\right)_{K} \geq 1$. Since $\left.\hat{\varphi}\right|_{K}=\varphi$, we get $\left(\left.\hat{\varphi}^{G}\right|_{K}, \varphi\right)_{K} \geq 1$, and using Lemma 2.1 again, we get $\left(\hat{\varphi}^{G}, \varphi^{G}\right)_{G} \geq 1$. Since we are assuming that the possibility (c) of the conclusion does not occur, we conclude that $\hat{\varphi}^{G}(1)=\left[G: T_{\varphi}\right]$ and $q-1$ are relatively prime. Hence $q-1|| T_{\varphi} \mid$ and so by a theorem of Philip Hall ([8] Theorem 9.3.1), some conjugate of $T K \leq T_{\varphi}$. Let $R$ denote the representation of $G / K$ acting by conjugation on the irreducible module $K$. If the representation is not faithful, then the kernel, say $N / K$ is either contained in $M / K$ or contains $M / K$. Since $M$ is not abelian, $N / K<M / K$. Therefore the character $\theta$ remains the same on $T K / K$ whether $K$ is considered as a $G / K$-module or as a $G / N$ module.

Hence, by Lemma $2.15,\left.R\right|_{T K / K}=t \cdot \rho_{T K / K}$, where $\rho_{T K / K}$ is the regular representation of $T K / K$ and $t$ is some positive integer. In every regular representation module, the dimension of the subspace of fixed points is 1 and so $T K / K$ fixes $r^{t}-1$ non-trivial elements of $K$. Since the size of $\rho_{T K / K}(1)=|T K / K|=|T|=q-1$, we get $|K|=r^{t(q-1)}$ and so there are $r^{t(q-1)}-1$ non-trivial elements in $K$. Since $G / K$ is Frobenius and since $|T K / K|=|M / K|-1, T K / K$ acts transitively on the non-trivial elements of $M / K$. Therefore, two different $T K / K$ 's generate all of $G / K$, and this implies two different $T K / K$ 's cannot fix the same non-trivial element in $K$, since $K$ 
is minimal normal and $M$ is non-abelian. There are $q$ conjugates of $T K / K$, each fixing $r^{t}-1$ non-trivial elements in $K$. Thus, equating the total number of fixed elements in $K$, we get

$$
q\left(r^{t}-1\right)=r^{t(q-1)}-1
$$

i.e.,

$$
q=\frac{r^{t(q-1)}-1}{r^{t}-1}=r^{t(q-2)}+r^{t(q-3)}+\cdots+1>2^{q-2} .
$$

This clearly implies that $q=3$. The proof is complete.

\section{Results}

The main theorem of this paper is the following:

Theorem 3.1. Suppose $G$ is a finite solvable group having exactly one irreducible character $\chi$ of degree $\chi(1)=m>1$ and every irreducible character $\eta$ of $G$ is such that $(\eta(1), m)=1$. Then $G$ is isomorphic to one of the following:

(a) An extra-special 2-group

(b) The group of all transformations $x \mapsto \alpha x+\beta(\alpha \neq 0)$ on a field of order $p^{n} \neq 2$

(c) $S_{4}$, the symmetric group on 4 letters

(d) $S L(2,3)$, the special linear group of $2 \times 2$ matrices over field of order 3

(e) A semidirect product of $S L(2,3)$ with $C_{3} \times C_{3}$.

The character $\chi$ is faithful in cases (a) and (b) and non-faithful in cases (c), (d), and (e).

The proof is carried out in a sequence of lemmas.

Lemma 3.2. Suppose the hypotheses of the theorem hold and that $\chi$ is faithful. Then $G$ is isomorphic to one of the groups listed in (a) and (b).

Proof. Let $\chi(1)=m=p_{1}^{m_{1}} \cdot p_{2}^{m_{2}} \cdots \cdots p_{k}^{m_{k}}$, where each $p_{i}$ is prime and let $N$ be a minimal normal subgroup of $G$. Then, by hypothesis, $B / N$ has no irreducible character $\zeta$ such that $p_{i} \mid \zeta(1)$ for any $i=1,2, \ldots, k$. By Lemma 2.4, $G / N$ has normal abelian $p_{i}$-Sylow subgroup for every $i$. Let $P_{i}$ be a $p_{i}$-Sylow subgroup of $G$ and $H$ be the group $P_{1} \cdot P_{2} \cdots P_{k} \cdot N$ so that $H$ is normal in $G$. 
Claim. $H=G$

Suppose $H \neq G$ and that $M$ is a maximal normal subgroup of $G$ containing $H$. Then $G / M$ is cyclic of order $p$ for some prime $p \neq p_{i}$ for any $i$. By Lemma 2.2 ,

$$
\left.\chi\right|_{M}=a \sum_{i=1}^{t} \theta^{g_{i}}
$$

where $\theta^{g_{i}}$ are irreducible characters of $M, a$ and $t$ are positive integers and $t=\left[G: T_{\theta}\right]$. Equating the degrees, we get

$$
\chi(1)=a \cdot t \cdot \theta(1) .
$$

If $\theta(1)=1$, then $M^{\prime} \subseteq \bigcap_{i=1}^{t} \operatorname{ker} \theta^{g_{i}} \subseteq \operatorname{ker} \chi=1$, and so $M$ is abelian. But then Lemma 2.6 implies $\chi(1) \mid[G: M]$, which is a contradiction. Therefore $\theta(1)>1$. Also, since $[G: M]$ is a prime number, $t=1$ or $[G: M]$. If $t=[G: M]$, then $\theta^{G}$ is irreducible (Lemma 2.3) of degree $\theta(1)[G: M]$, and since $\theta(1) \mid \chi(1)$, this is a contradiction to our hypothesis. Therefore $t=1$. By Lemma 2.11, $\theta$ extends to an irreducible character $\hat{\theta}$ of $G$. By hypothesis, $\hat{\theta}=\chi$ and so $\left.\chi\right|_{M}=\theta$. But then $\theta=\left.\chi\right|_{M}$ has $[G: M]$ distinct extensions, once again contradicting our hypothesis. This proves our claim that $H=G$.

Since $N$ is normal and abelian, Lemma 2.6 and our hypotheses imply that $\chi$ is the only non-linear irreducible character of $G$. Lemma 2.14 yields the required result.

\section{Remark 3.3.}

(i) As seen in the above proof, the hypothesis of Lemma 3.2 implies that $\chi$ is the only non-linear irreducible character of $G$.

(ii) If $G$ is an extra-special 2-group of order $2^{k}$, then $\chi(1)=2^{(k-1) / 2}$, so that the degree of $\chi$ determines the order of the group and conversely.

(iii) If $G$ is a group of all transformations $x \mapsto \alpha x+\beta(\alpha \neq 0)$ on a field of order $p^{n} \neq 2$, then $G$ is a Frobenius group of order $p^{n}\left(p^{n}-1\right)$. If $M$ is the Frobenius kernel of $G$, then $|M|=p^{n}, M$ is elementary abelian and $G / M$ is cyclic. The degree of $\chi$ is $p^{n}-1$ and so once again the order of $G$ is determined by the degree of $\chi$, and conversely.

We now set out to investigate ker $\chi$.

Lemma 3.4. Suppose that the hypothesis of Theorem 3.1 holds and that ker $\chi=K>1$. Then $G / K$ is a Frobenius group of the type listed in (b) of Theorem 3.1. If $M / K$ denotes the Frobenius kernel, then $M^{\prime}=K$. 
Proof. By Lemma 3.2, $G / K$ is either an extra special 2-group or a Frobenius group. We shall prove $G / K$ cannot be a 2 -group. To this end, assume $G$ is a minimal counter-example so that $G / K$ is a 2-group. By minimality of $|G|$, $K$ is a minimal normal subgroup of $G$. Then Lemma 2.6 and the hypothesis of Theorem 3.1 together imply that $\chi$ is the only non-linear irreducible character of $G$. Therefore, by Lemma 2.14, $G$ is either an extra-special 2-group or a Frobenius group, and in either case $\chi$ is faithful. This contradicts our assumption that $\operatorname{ker} \chi=K>1$. Hence, $G / K$ is a Frobenius group.

It remains to prove $M^{\prime}=K$, where $M / K$ is the Frobenius kernel of $G / K$. Clearly $M^{\prime} \leq K$, since $M / K$ is abelian (see Remark 3.3 (iii)). If $M^{\prime}<K$, then $M / M^{\prime}$ is a normal, abelian subgroup of $G / M^{\prime}$. Hence it follows once again by Lemma 2.6 and the hypotheses (note $\chi(1)=[G: M]$ ) that $\chi$ is the only non-linear character of $G$. In such a case $\chi$ is always faithful and again we have a contradiction. This proves Lemma 3.4.

As we will see later, the following lemma and Lemma 2.6 together reduce the proof to the case $\chi(1)=2$ or 3 .

Lemma 3.5. Suppose the hypotheses of Theorem 3.1 hold and that ker $\chi=K>1$. Further, suppose that $K$ is minimal normal and that $M$ (same as in Lemma 3.4) is a $p$-group for some prime $p$. Then $G \cong S L(2,3)$.

Proof. The proof is carried out in 3 steps.

Step $1 . K \leq Z(G)$.

Suppose $K \not \leq Z(G)$. Let $|M / K|=p^{n}$ so that $[G: M]=\chi(1)=p^{n}-1$. Since $G$ is solvable, we can write $G=M T$ where $T \cong G / M$ is a cyclic group of order $p^{n}-1$. Since $K$ is minimal normal and $M$ is a $p$-group, we have $K \leq Z(M)$. Then $G / C_{G}(K)$ is cyclic; say $G / C_{G}(K)=\langle\bar{g}\rangle$, where $\bar{g}=g C_{G}(K)$, $g \in T$. The group $\langle g\rangle$ acts on the irreducible characters of $K$ and if $g$ fixes every irreducible character of $K$, then by Lemma 2.7 (applied to the character table of $K) g \in C_{G}(K)$, contrary to our assumption that $G \neq C_{G}(K)$. So there exists $\varphi \neq 1$, an irreducible character of $K$, such that $\phi \neq \phi^{g}$. Let $\eta$ be an irreducible character of $M$ such that $\left(\phi^{M}, \eta\right)_{M} \geq 1$. If $\eta(1)=1$, then $\eta=1$ on $M^{\prime}=K$ and by Lemma 2.1 , we get $\left(\phi^{M}, \eta\right)_{M}=(\phi, 1)_{K} \geq 1$. This is impossible since $\phi \neq 1$. Therefore $\eta(1)>1$. Since $K \leq Z(M),\left.\eta\right|_{K}=a \cdot \phi$ for some positive integer $a$ and so $\left.\eta^{g}\right|_{K}=a \cdot \phi^{g}$. Hence, $\eta \neq \eta^{g}$ and so $T_{\eta}$, the stabilizer of $\eta$, is a proper subgroup of $G$. If $T_{\eta}=M$, then $\eta^{G}$ would be an irreducible character (Lemma 2.3) of degree $\chi(1) \eta(1)>\chi(1)$ (since $\eta(1)>1$ ). This is impossible by our hypotheses. Thus $G>T_{\eta}>M$. Let $\theta$ be an irreducible character of $G$ 
such that $\left(\eta^{G}, \theta\right)_{G} \geq 1$. By Lemma 2.10, there exists an irreducible character $\zeta$ of $T_{\eta}$ such that

(i) $\zeta^{G}=\theta$ and

(ii) $\left.\zeta\right|_{M}=r \cdot \eta$ for some positive integer $r$.

Then the equation (i) implies $(\theta(1), \chi(1)) \neq 1$. Therefore $\theta=\chi$, by hypothesis, and so $\chi(1)=p^{n}-1=\zeta^{G}(1)=\zeta(1)\left[G: T_{\eta}\right]$. Hence

$$
\zeta(1)=\frac{p^{n}-1}{\left[G: T_{\eta}\right]} .
$$

On the other hand the equation (ii) implies $\zeta(1)=r \cdot \eta(1)$. Since $p \mid \eta(1)$ ( $\eta$ is an irreducible character of $M$, a $p$-group), we get $p \mid \zeta(1)$ and this is a contradiction to (1). Hence $K \leq Z(G)$.

Step 2. $Z(M)=K$.

If $Z(M)>K$, then $G / Z(M)$ is a Frobenius group with kernel $M / Z(M)$ and complement a cyclic group of order $p^{n}-1$. But an element of order $p^{n}-1$ cannot act fixed-point free on a group of order $<p^{n}$ and $|M / Z(M)|<p^{n}$. Therefore $Z(M)=K$.

Step 3. We now prove Lemma 3.5.

Since $K$ is minimal normal and contained in $Z(G),|K|=p$ for some prime p. Also $M$ is a class 2, $p$-group with $Z(M)=K=M^{\prime}$ (Lemma 3.4) and $M / K$ is elementary abelian. Thus $M / K$ is a vector space over $K$, considered as a field of order $p$. Then the map from $M / K \times M / K \rightarrow K$ defined by $[a K, b K] \mapsto$ $a^{-1} b^{-1} a b$ is easily checked to be a well-defied alterating non-degenerate linear form on $M / K \times M / K$. Thus $M / K$ is a symplectic space. The cyclic group $T$ of order $p^{n}-1$ can be considered a subgroup of $\operatorname{Sp}(2 \cdot n / 2, p)$ (the group of symmetries of the space $M / K)$. Since $T$ acts irreducibly on $M / K$, by a theorem on symplectic groups ([9] Satz 9.23, p.228) we get $|T|=p^{n}-1 \mid p^{n / 2}+1$. This implies $p=n=2$. Hence, $|K|=2,|M / K|=4$ and $|G / M|=3$, i.e., $G$ is a group of order 24 and $\chi(1)=3$. It is now easy to check that $G \cong \operatorname{SL}(2,3)$.

The next two lemmas are particular cases of Theorem 3.1 and settle cases when $\chi(1)=2$ or 3 .

Lemma 3.6. Suppose the hypotheses of Theorem 3.1 hold with $\chi(1)=2$. If $\operatorname{ker} \chi=K>1$, then $G \cong S_{4}$. 
Proof. From Lemma 3.4, we have the following picture:

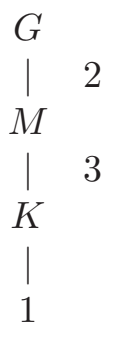

where $M^{\prime}=K$ and $G / K \cong S_{3}$.

The proof is carried out in a sequence of steps.

Step 1. $M$ is a Frobenius group with kernel $K$.

By Lemma 2.8, it is enough to show that every non-trivial irreducible character $\varphi$ of $K$ is such that $\varphi^{M}$ is an irreducible character of $M$. Let $\eta$ be an irreducible character of $M$ such that $\left(\eta, \varphi^{M}\right)_{M} \geq 1$. Lemma 2.1 and the fact that $M^{\prime}=K$ imply that $\eta(1)>1$. Then $T_{\eta}=G$, for if $T_{\eta}=M$, then $\eta^{G}$ would be an irreducible character of $G$ with $2 \mid \eta^{G}(1)$ and $2<\eta^{G}(1)$, contrary to the hypothesis. Thus, Lemma 2.13 applies and we have the following possibilities.

(i) $\left.\eta\right|_{K}$ is irreducible,

(ii) $\left.\eta\right|_{K}=a \varphi, a^{2}=[M: K]$,

(iii) $\left.\eta\right|_{K}=\sum_{i=1}^{3} \varphi_{i}, \varphi_{i}$ distinct irreducible characters of $K$ transitively permuted by $M\left(\varphi_{1}=\varphi\right.$, say $)$.

Possibility (ii) is ruled out since $a^{2}$ cannot be 3 . Suppose possibility (i) occurs. Since $T_{\eta}=G$, by Lemma 2.12, $\eta$ extends to an irreducible character $\hat{\eta}$ of $G$. Then the characters $\left\{\omega \cdot \hat{\eta} \mid \omega\right.$ irreducible character of $\left.G / K \cong S_{3}\right\}$ are all irreducible by Lemma 2.9. But one of the $\omega$ 's is $\chi$ and $\hat{\eta}(1)>1$, and this is a contradiction to our hypothesis. Therefore, only the possibility (iii) can occur and this is equivalent to saying $\varphi^{M}=\eta$ (Lemma 2.3). The proof of Step 1 is complete.

Step 2. $K$ is an abelian 2-group.

We first note that $K$ is nilpotent since it is the kernel of a Frobenius group ([7] Theorem 10.3.1 (iii), p.339). Next we prove the following.

Claim. $|K|$ is even. 
Proof. Suppose $|K|$ is odd and $G$ is a minimal counterexample. If there is a normal subgroup $L$ of $G$ such that $1<L<K$, then by minimality of $G, 2 \mid[K: L]$, contrary to our supposition that $|K|$ is odd. Therefore we can assume $K$ is minimal normal in $G$ and $K \cong C_{P} \times C_{p} \times \cdots \times C_{p}$, with $p>3$ $(p \neq 3$ since $M$ is Frobenius with kernel $K)$. Thus, we can regard $K$ as an irreducible module for $G / K \cong S_{3}$. Since $p>3$, the degree of an irreducible representation of $S_{3}$ over $C_{p}$ is either 1 or 2 . Also, $K \neq C_{p}$, since $\operatorname{Aut}\left(C_{p}\right)$ is cyclic and $G / K$ is a non-cyclic group acting faithfully on $K$. Therefore, we can assume $K \cong K_{1} \times K_{2}$ with $K_{1} \cong K_{2} \cong C_{p}$. Let $t K$ and $x K$ be elements of order 2 and 3 respectively in $G / K$. The minimal polynomial of $t K$ on $K$ is $Y^{2}-1=(Y+1)(Y-1)$, for if $t K$ acts as either 1 or -1 on $K$, then $t K$ and $x K$ commute on $K$, so that $\langle[t K, x K]\rangle=\langle x K\rangle$ acts trivially on $K$ and this is not the case. Therefore, we may suppose $\left.t K\right|_{K_{1}}=-1$ and $\left.t K\right|_{K_{2}}=1$. Let $\zeta \neq 1_{K}$ be an irreducible character of $K / K_{2}$. Then $\zeta \neq \zeta^{t}$ and we know $\zeta, \zeta^{x}, \zeta^{x^{2}}$ are distinct since $M$ is Frobenius. Let $g \neq 1 \in K_{2}$ and let $g^{x^{-1}}=g_{1} \cdot g_{2}$ for some $g_{1} \neq 1 \in K_{1}$ and $g_{2} \in K_{2}$. (If $g_{1}=1$, then $K_{2}$ would be normal in $G$ whereas $K$ is minimal normal in $G)$. Then $\zeta^{t}(g)=\zeta\left(g^{t}\right)=1$ whereas $\zeta^{x}(g)=\zeta\left(g^{x^{-1}}\right)=\zeta\left(g_{1}\right) \zeta\left(g_{2}\right)=\zeta\left(g_{1}\right) \neq 1$. Thus $\zeta^{x} \neq \zeta^{t}$. Similarly, $\zeta^{x^{2}} \neq \zeta^{t}$ and hence, there are more than 3 conjugates of $\zeta$. It now follows from Lemma 2.3 that $\zeta^{G}$ is irreducible. This is a contradiction to our hypothesis on the degrees of the irreducible characters of $G$ and the proof of the claim is complete.

Since $K$ is nilpotent, we can write $K=P_{1} \times P_{2} \times \cdots \times P_{r}$ with $P_{1}$ a 2-Sylow subgroup of $K$. Considering the quotient group $G / P_{1}$ and using induction on $|G|$, we obtain $P_{i}=1$ for $i>1$. Thus, $K=P_{1}$, a 2-group. If $K$ is not abelian, then there exists an irreducible character $\theta$ of $K$ such that $\theta(1)=2^{\ell}, \ell \geq 1$. Then $\theta^{M}$ is irreducible since $M$ is Frobenius. If $T_{\theta^{M}}=M$, then $\left(\theta^{M}\right)^{G}=\theta^{G}$ is irreducible by Lemma 2.3, and if $T_{\theta^{M}}=G$, then $\theta^{M}$ extends to an irreducible character of $G$ by Lemma 2.11. In either case, we have a contradiction to our hypothesis. Therefore $K$ is an abelian 2-group. The proof of Step 2 is complete.

Step 3. $K \cong C_{2} \times C_{2}$.

Let $\Omega_{1}(K)=\left\{g \in K \mid g^{2}=1\right\}$. Then, $1<\Omega_{1}(K)$ is a characteristic subgroup of $K$, and so $\Omega_{1}(K) \triangleleft G$. Using induction on $|G|$, we conclude that

$$
\text { either } K / \Omega_{1}(K) \cong C_{2} \times C_{2} \text {, or } K=\Omega_{1}(K)
$$

Since $x$ (same as in Step 2) does not fix anything in $\Omega_{1}(K), \Omega_{1}(K)$ decomposes 
into $\Omega_{1}(K)=L_{1} \times L_{2} \times \cdots \times L_{r}$ with each $L_{i} \cong C_{2} \times C_{2}$ and is $\langle x\rangle$-irreducible.

Claim. $L_{i}$ is $\langle t\rangle$-invariant for every $i$.

Proof. Fix $i$ and suppose $L_{i} \neq L_{i}^{t}$. By the Frattini argument, $N_{G}(\langle x\rangle) \cdot M=$ $G$. Since $M=\langle x\rangle K$, we obtain $N_{G}(\langle x\rangle) \cdot K=G$. Also, since $\langle x\rangle \cdot K$ is Frobenius, $N_{G}(\langle x\rangle) \cap K=1$. Thus, we may suppose that $t \in N_{G}(\langle x\rangle)$, and so $x$ leaves $L_{i}^{t}$ invariant. Therefore, $L_{i} \cap L_{i}^{t}=1$, for otherwise $L_{i} \cap L_{i}^{t}$ would be an $\langle x\rangle$-invariant subgroup of order 2. But then, $x$ fixes some non-trivial element of $K$. Hence we may suppose that $L_{i}^{t}=L_{j}$ for some $j$, i.e., $\Omega_{1}(K)=L_{i} \times L_{j} \times \prod_{s \neq i, j} L_{s}$ with $L_{i}^{t}=L_{j}$. Let $L_{i}=\langle a\rangle \times\langle b\rangle, a^{2}=b^{2}=1$. Let $\varphi \neq 1$ be an irreducible character of $\Omega_{1}(K)$ having $\langle b\rangle \times L_{j} \times \prod_{s \neq i, j} L_{s}$ as its kernel. Then $\varphi, \varphi^{x}, \varphi^{x^{2}}$ are distinct since $\langle x\rangle \cdot \Omega_{1}(K)$ is Frobenius. Also, since $L_{j}$ is $\langle x\rangle$-invariant, $L_{j}$ is contained in the kernel of each $\varphi, \varphi^{x}$ and $\varphi^{x^{2}}$, whereas $\varphi^{t}\left(a^{t}\right)=\varphi(a) \neq 1$. Therefore $\varphi^{t} \neq \varphi, \varphi^{x}, \varphi^{x^{2}}$. Since $K$ is abelian, $\varphi$ extends to an irreducible character $\hat{\varphi}$ of $K$ (Lemma 2.12) and the above argument implies $T_{\hat{\varphi}}=K$. Hence $\hat{\varphi}^{G}$. is irreducible (Lemma 2.3) of degree 6. This contradiction proves the claim.

Thus $L_{i} \triangleleft G$ for every $i$. Then, by induction on $|G|, G / L_{1} \cong S_{4}$ or $S_{3}$. This, together with (2), implies either (i) $K / \Omega_{1}(K) \cong C_{2} \times C_{2}$ with $\Omega_{1}(K) \cong C_{2} \times C_{2}$, or (ii) $K=\Omega_{1}(K) \cong C_{2} \times C_{2}$, or (iii) $K=\Omega_{1}(K) \cong\left(C_{2} \times C_{2}\right) \times\left(C_{2} \times C_{2}\right)$ with each $\left(C_{2} \times C_{2}\right) \triangleleft G$. Thus, we conclude that one of the following holds.

(i) $K \cong C_{2} \times C_{2}$

(ii) $K \cong\left(C_{2} \times C_{2}\right) \times\left(C_{2} \times C_{2}\right)$ with each $\left(C_{2} \times C_{2}\right) \triangleleft G$

(iii) $K \cong C_{4} \times C_{4}$

If (i) holds, we are done. We shall prove that neither (ii) nor (iii) can hold. To this end, first suppose (ii) holds and let $K=L_{1} \times L_{2}$ where $L_{i} \cong C_{2} \times C_{2}$ and $L_{i} \triangleleft G$ for $i=1,2$. As before, if $t$ is an element of order 2 in $N_{G}(\langle x\rangle)$, then $t$ cannot centralize either $L_{1}$ or $L_{2}$; for if it does, say $L_{1}$, then $C_{G}\left(L_{1}\right)$ would be a normal subgroup of $G$ contaiing $K$ and $3 \mid\left[G: C_{G}\left(L_{1}\right)\right]$. Since $G / K \cong S_{3}$, $C_{G}\left(L_{1}\right)=G$, contradicting the fact that $M$ is Frobenius. Therefore, there exists an irreducible character say $\zeta \neq 1$ of $L_{1}$ such that $\zeta^{t} \neq \zeta$ (Lemma 2.7). Actually we can produce an irreducible character $\theta$ of $L_{1}$ such that $\theta^{t} \neq \theta$ and also $\theta^{t} \neq \theta^{x}$. to get such a $\theta$, first we get a $\zeta$, as above. If $\zeta^{t} \neq \zeta^{x}$, then we set $\theta=\zeta$. If $\zeta^{t}=\zeta^{x}$, then we let $\theta=\zeta^{t}$. Then $\left.\theta^{t}=\left(\zeta^{t}\right)^{t}\right)=\zeta \neq \theta$ and $\theta^{x}=\left(\zeta^{t}\right)^{x}=\left(\zeta^{x}\right)^{x}=\zeta^{x^{2}} \neq \zeta=\theta^{t}$. Similarly, we can obtain an irreducible character $\varphi$ of $L_{2}$ such that $\varphi^{t} \neq \varphi$ and also $\varphi^{t} \neq \varphi^{x^{2}}$. Then $\theta \cdot \varphi$ is an irreducible character of $K \cong L_{1} \times L_{2}$ ([7] Theorem 3.7.1, p.100). We now check 
that $\theta \cdot \varphi$ has 6 conjugates. Clearly, $\theta \cdot \varphi,(\theta \cdot \varphi)^{x}$ and $(\theta \cdot \varphi)^{x^{2}}$ are distinct since $M$ is Frobenius. $(\theta \cdot \varphi)^{t}=\theta \cdot \varphi$ implies $\theta^{t}=\theta$ and $\varphi^{t}=\varphi$, a contradiction. $(\theta \cdot \varphi)^{t}=(\theta \cdot \varphi)^{x}$ implies $\theta^{t}=\theta^{x}$, a contradiction. $(\theta \cdot \varphi)^{t}=(\theta \cdot \varphi)^{x^{2}}$ implies $\varphi^{t}=\varphi^{x^{2}}$, a contradiction. Hence $(\theta \cdot \varphi)^{G}$ is an irreducible character of $G$ (Lemma 2.3) of degre 6. This is a contradiction to our hypothesis. Therefore (ii) cannot hold.

Now suppose (iii) holds, i.e. $K \cong C_{4} \times C_{4}$, and $\Omega_{1}(K)=C_{2} \times C_{2} \triangleleft G$. Then $t$ does not centralize $\Omega_{1}(K)$ since $G / K$ has no normal subgroup of index 3. Therefore, we may suppose $\Omega_{1}(K)=\langle a\rangle \times\langle b\rangle, a^{2}=b^{2}=1$ and $a^{t}=b$, $b^{t}=a$. Let $h \in K$ be such that $h^{2}=a,|h|=4$. Then, $\langle h\rangle \cap\left\langle h^{t}\right\rangle=1$, since $h^{2}=a$ is not fixed by $t$. Let $K_{\circ}=\left\langle h h^{t}\right\rangle$. Then $\left|K_{\circ}\right|=4$ and $t$ centralizes $K_{\circ}$. Also $\left(h K_{\circ}\right)^{t}=h^{t} K_{\circ}=h^{-1} K_{\circ}$. If $\varphi \neq 1$ is a faithful irreducible character of $K / K_{\circ}$ then $\varphi^{t} \neq \varphi$ and $\operatorname{ker} \varphi^{t}=\operatorname{ker} \varphi$. Moreover $\left.\mid K_{\circ} \cdot \Omega_{1}(K) / \Omega_{(} K\right) \mid=2$ and so $x$ cannot normalize $K_{\circ} \Omega_{1}(K) / \Omega_{1}(K)$, since $G / \Omega_{1}(K) \cong S_{4}$, and $M / \Omega_{1}(K)$ is Frobenius. Therefore, $K_{\circ}$ is not normalized by $x$, and so $K_{\circ}, K_{\circ}^{x}, K_{\circ}^{x^{2}}$ are distinct. Hence, $\varphi, \varphi^{x}, \varphi^{x^{2}}$ are distinct and none of them equals $\varphi^{t}$ since the kernels are different. This implies, again, that $\varphi$ has 6 conjugates and so $\varphi^{G}$ is an irreducible character of $G$ of degree 6. This contradiction proves Step 3 and hence the lemma.

Lemma 3.7. Suppose the hypothesis of Theorem 3.1 holds with $\chi(1)=3$. If $\operatorname{ker} \chi=K>1$ then $G$ is isomorphic to either $S L(2,3)$ or to a semidirect product of $S L(2,3)$ with $C_{3} \times C_{3}$.

Proof. From Lemma 3.4, we have the following picture:

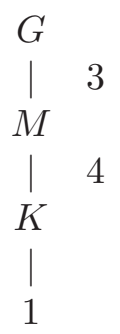

with $M^{\prime}=K$ and $G / K \cong A_{4}$.

Once again the proof is carried out in a sequence of steps.

Step 1. $M$ has a normal 2-complement, say $H$.

By Lemma 2.5, it is enough to show that $2 \mid \theta(1)$ for every non-linear irreducible character $\theta$ of $M$. To see this, we first observe that $T_{\theta}=G$, for 
if $T_{\theta}=M$, then by Lemma $2.3, \theta^{G}$ would be an irreducible character of $G$ of degree divisible by 3 and $>3$. This is a contradiction to the hypotheses. Lemma 2.13 now yields the following three possibilities:

(i) $\left.\theta\right|_{K}$ is irreducible

(ii) $\left.\theta\right|_{K}=a \varphi$ for some irreducible character $\varphi$ of $K$ and $a^{2}=[M: K]=4$

(iii) $\left.\theta\right|_{K}=\sum_{i=1}^{4} \varphi_{i}, \varphi_{i}$ distinct irreducible characters of $K$

If (i) occurs, then since $[G: M]$ is prime and $\theta$ is stabilizied by $G, \theta$ extends to an irreducible character say $\hat{\theta}$ of $G$ by Lemma 2.11. Thus $\left.\theta\right|_{K}$ as an irreducible character of $K$ extends to an irreducible character $\hat{\theta}$ of $G$ and is stabilized by $G$. We can now apply Lemma 2.9 and get $\left(\left.\theta\right|_{K}\right)^{G}=\sum_{\omega} \omega(1) \omega \hat{\theta}$ where $\omega$ ranges over all irreducible characters of $G / K$ and the characters $\{\omega \hat{\theta}\}$ are distinct and irreducible. Since $G / K$ is $A_{4}$, and $\hat{\theta}$ is non-linear, one of $\omega \hat{\theta}$ is an irreducible character of degree $>3$ and divisible by 3 . This is a contradiction to the hypothesis. Thus, only either (ii) or (iii) can occur and in either case, clearly, $2 \mid \theta(1)$. This proves Step 1.

Step 2. If the normal 2-complement $H=1$, so that $M$ is a 2-group, then $G$ is isomorphic to $\mathrm{SL}(2,3)$.

Let $K=K_{0} \triangleright K_{1} \triangleright K_{2} \triangleright K_{3} \cdots \triangleright K_{n} \triangleright 1$ be a part of a chief series for $G$. Then $K_{n} \cap Z(M) \neq 1$, since $M$ is a 2-group, and so the minimality of $K_{n}$ implies $K_{n} \leq Z(M)$. Therefore every subgroup of $K_{n}$ is normal in $M$. Let $g$ be an element of order 3 in $G$ so that $G=\langle g\rangle \cdot M$. Since $|g|=3$, under the action of $g$ each irreducible consituent of $K_{n}$ has order equal to either 2 or 4 . But then, every such constituent of $K_{n}$ is normal in $G$. Therefore $\left|K_{n}\right|=2$ or 4. The same argument applied to th group $G / K_{n}$ shows that $\left|K_{n-1} / K_{n}\right|=2$ or 4, etc. Thus, $\left|K_{i} / K_{i+1}\right|=2$ or $4 \forall i$. Suppose $\left|K_{n}\right|=4$, so that $K_{n} \cong C_{2} \times C_{2}$. Then $g$ does not centralize any non-trivial element of $K_{n}$ and so there exists a linear character $\zeta \neq 1$ of $K_{n}$ such that $\zeta \neq \zeta^{g}$ (Lemma 2.7). Let $\varphi$ be an irreducible character of $M$ such that $\left(\zeta^{M}, \varphi\right)_{M} \geq 1$. Then Frobenius reciprocity (Lemma 2.1) and the fact that $K_{n} \leq K=M^{\prime}$ imply that $\varphi(1)>1$. Since $K_{n} \leq Z(M)$, we conclude that $\left.\varphi\right|_{K_{n}}=a \cdot \zeta$ for some positive integer $a$, and so $\left.\varphi^{g}\right|_{K_{n}}=a \cdot \zeta^{g} \neq\left.\varphi\right|_{K_{n}}$. Thus, $\varphi^{g} \neq \varphi$, and so $T_{\varphi}<G$. Therefore $T_{\varphi}=M$, since $[G: M]$ is prime. But then, Lemma 2.3 implies $\varphi^{G}$ is irreducible. This is a contradiction since $\varphi^{G}(1)=3 \cdot \varphi(1)>3$. Hence $\left|K_{n}\right|=2$. Applying the same argument to $G / K_{n}$, we obtain $\left|K_{n-1} / K_{n}\right|=2$, etc. Thus $\left|K_{i} / K_{i+1}\right|=2$ $\forall i$, and $g$ centralizes $K_{i} / K_{i+1}$ for every $i$. This implies that $g$ centralizes $K$ 
since $(|g|,|K|)=1$. Also, $G^{\prime}=M$, for clearly, $G^{\prime} \subseteq M$ and $G^{\prime} \supseteq M^{\prime}=K$ and $G / K$, being isomorphic to $A_{4}$, does not have any proper normal subgroups between $M$ and $K$. Since $g \in C_{G}(K), G / C_{G}(K)$ is a 2-group. This implies that $G=C_{G}(K)$, as $M$ is 2-Sylow and contained in $G^{\prime}$. It now follows that $Z(M)=K$, since $G / K$ is isomorphic to $A_{4}$. Thus $M$ is a class 2, 2-group. Also, $M^{\prime}$ is generated by commutators $[x, y]$, where $x$ and $y$ range over the coset representatives of $Z(M)$ in $M$. Let $M=K \cup K x \cup K y \cup K x y$. Then, $M^{\prime}=\langle[x, y],[x, x y],[y, x y]\rangle$. But, $[x, x y]=[x, x][x, y]$ and $[y, x y]=[y, x][y, y]$, since $M$ is a class 2-group. Therefore $M^{\prime}=\langle[x, y]\rangle$ is a cyclic 2-group. Also, $[x, y][x, y]=\left[x, y^{2}\right]=1$, since $y^{2} \in K=Z(M)$. Hence, $|K|=2$ and so $|G|=24$. This clearly implies that $G$ is isomorphic to $\mathrm{SL}(2,3)$.

From now on, we suppose $H>1$.

Step 3. We may asume $[K: H]=2$.

Since $H$ is the normal 2-complement in $M$ and since $K$ is a normal subgroup of $M$ of index 4 in $M$, we get $H \subset K$. By using induction on $|G|$, we obtain either $G / H$ is isomorphic to $A_{4}$, so that $K=H$, or $G / H$ is isomorphic to $\operatorname{SL}(2,3)$, so that $[K: H]=2$, or $G / H$ is isomorphic to $\operatorname{SL}(2,3) \cdot\left(C_{3} \times C_{3}\right)$. The last possibility cannot occur since $M / H$ is a 2-group. Thus it is enough to show that $K \neq H$. Suppose $K=H$. Then, $H$ is minimal normal in $G$, for if $L<H$ and $L \triangleleft G$, then by induction $G / L$ is isomorphic to either $\operatorname{SL}(2,3)$ or $\mathrm{SL}(2,3) \cdot\left(C_{3} \times C_{3}\right)$ and so in either case 2||$H \mid$, contrary to the fact that $H$ is a $2^{\prime}$-group. Therefore, $H=C_{p} \times C_{p} \times \cdots C_{p}$ for some odd prime $p$. We now claim $M$ is a Frobenius group with kernel $H$. Let $\varphi \neq 1$ be a linear character of $H$. By Lemma 2.8, it is enough to show $\varphi^{M}$ is irreducible. To this end, first consider the case $T_{\varphi}=G$. In particular, $M$ stabilizes $\varphi$ and so by Lemma 2.12, $\varphi$ extends to a linear character $\hat{\varphi}$ of $M$. But then, since $M^{\prime}=K$ (Lemma 3.4) and $K=H$, we get $\left.\hat{\varphi}\right|_{H}=1_{H}$ whereas $\left.\hat{\varphi}\right|_{H}=\varphi \neq 1_{H}$. Therefore $T_{\varphi}<G$. Suppose $3 \mid\left[G: T_{\varphi}\right]$ and let $\eta$ be an irreducible character of $G$ such that $\left(\varphi^{G}, \eta\right)_{G} \geq 1$. By Lemma 2.10, there exists an irreducible character $\zeta$ of $T_{\varphi}$ such that $\zeta^{G}=\eta$. Since $3 \mid \zeta^{G}(1)$, we get $\eta=\chi$. So by Lemma 2.1, $\left(\varphi^{G}, \chi\right)_{G}=\left(\varphi,\left.\chi\right|_{H}\right)_{H} \geq 1$. This is impossible since $\left.\chi\right|_{H}=3 \cdot 1_{H}$. Thus, we have $T_{\varphi}<G$ and $3 \nmid\left[G: T_{\varphi}\right]$. We can write $M=R \cdot H, R \cap H=1$, where $R$ is a 2-Sylow subgroup of $M$ and $R$ is isomorphic to $C_{2} \times C_{2}$. Let $g H$ be an element of order 3 in $G / H$. Then since $G / H$ has no subgroup of index 2 , and since $3 \nmid\left[G: T_{\varphi}\right]$, we conclude that $T_{\varphi}$ is a conjugate of $\langle g\rangle H$. Thus $T_{\varphi} \cap R=1$, and so by Lemma $2.3, \varphi^{M}$ is irreducible, proving our claim that $M$ is Frobenius with kernel $H$. But then, since a 2-Sylow subgroup of a Frobenius complement 
is either cyclic or generalized quaternion, we cannot have $R$ being isomorphic to $C_{2} \times C_{2}$. This contradiction proves that $K \neq H$ and Step 3 is complete.

Step 4. We can now assume $1<H<K$ and $[K: H]=2$, because of Step 2 and Step 3. Then $G$ is isomorphic to $\mathrm{SL}(2,3) \cdot\left(C_{3} \times C_{3}\right)$.

First we claim that $H$ is an abelian $p$-group for some prime $p$. If $H$ is minimal normal in $G$ this is certainly true. So, suppose $H$ is not minimal normal in $G$ and let $L<H$ with $L \triangleleft G$. By induction on $|G|$, we may assume $G / L$ is isomorphic to $\mathrm{SL}(2,3) \cdot\left(C_{3} \times C_{3}\right)$ and that $L$ is minimal normal. We have the following picture:

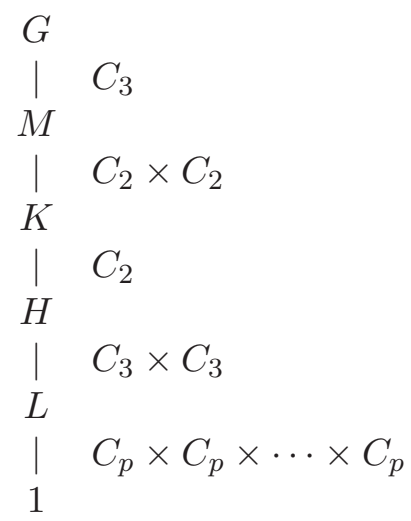

with $G^{\prime}=M$ and $G / H \cong \operatorname{SL}(2,3)$.

Suppose that $H$ is not abelian and let $\theta$ be a non-linear irreducible character of $H$. By Ito's Theorem (Lemma 2.6) $3 \mid \theta(1)$. Let $\varphi$ be an irreducible character of $G$ such that $\left(\theta^{G}, \varphi\right)_{G} \geq 1$. This implies by Lemma 2.1, $\left(\theta,\left.\varphi\right|_{H}\right)_{H} \geq 1$. Hence by Lemma 2.2, $\varphi(1)=r \cdot \theta(1)$ for some positive integer $r$. Therefore by hypothesis, $\varphi=\chi, r=1$ and $\left.\chi\right|_{H}=\theta$. This is impossible since $\left.\chi\right|_{H}=$ $3 \cdot 1_{H}$, whereas $\theta \neq 3 \cdot 1_{H}$ by our choice of $\theta$. Therefore $H$ is abelian. Let $L=C_{p} \times C_{p} \times \cdots \times C_{p}$. If $p \neq 3$, then $H=C_{3} \times C_{3} \times C_{p} \times C_{p} \times \cdots \times C_{p}$. By considering the smaller group $G /\left(C_{3} \times C_{3}\right)$ and using induction hypothesis, we get $H /\left(C_{3} \times C_{3}\right)$ is isomorphic to $C_{3} \times C_{3}$. So we may assume $p=3$ and that $H$ is an abelian 3 -group. This proves the claim that $H$ is an abelian $p$ group for some prime $p$. Next we claim that $3 \nmid\left[G: T_{\zeta}\right]$ for any irreducible character $\zeta \neq 1$ of $H$. To see this, let $\varphi$ be any irreducible character of $G$ such that $\left(\zeta^{G}, \varphi\right)_{G} \geq 1$. So $\left(\zeta,\left.\varphi\right|_{H}\right)_{H} \geq 1$ by 2.1. This in turn implies by 2.2 , $\left.\varphi\right|_{H}=a \sum_{i=1}^{t} \zeta^{g_{i}}$ where $t=\left[G: T_{\zeta}\right]$. If $3 \mid t$, then $3 \mid \varphi(1)$, and so $\varphi=\chi$. But then, $3 \cdot 1_{H}=\left.\chi\right|_{H}=\zeta^{g_{1}}+\zeta^{g 2}+\zeta^{g 3}$; a contradiction to the linear independence of irreducible characters. 
Thus, given any irreducible character $\zeta \neq 1$ of $H$, there is a 3-Sylow subgroup of $G / H(\cong \mathrm{SL}(2,3))$ stabilizing $\zeta$. There are 43 -Sylow subgroups of $G / H$ and any two of them generate $G / H$. So if two distinct 3-Sylow subgroups of $G / H$ stabilize the same character $\zeta \neq 1$ of $H$, then $G$ stabilizes $\zeta$. In particular, $M$ stabilizes $\zeta$, and by Lemma 2.12, $\zeta$ extends to a linear character $\hat{\zeta}$ of $M$. But then, $M^{\prime}=K \supset H$ and so $\left.\hat{\zeta}\right|_{H}=\zeta=1_{H}$, whereas $\zeta \neq 1_{H}$. Thus no two distinct 3-Sylow subgroups of $G / H$ stabilize the same irreducible character of $H$. Moreover, since the 3-Sylow subgroups in $G / H$ are conjugate, the number of irreducible characters of $H$ fixed by any one 3-Sylow subgroup of $G / H$ is the same as the number of irreducible characters of $H$ fixed by any other 3-Sylow subgroup of $G / H$. By Brauers' Lemma (2.7), the number of irreducible characters of $H$ fixed by a 3-Sylow subgroup of $G / H$ is equal to the number of elements of $H$ fixed by it. Also, the set of elements of $H$ fixed by a 3-Sylow subgroup of $G / H$ form a subgroup of $H$. Thus if $|H|=p^{n}$, and if the number of elements fixed by a 3-Sylow subgroup of $G / H$ is $p^{n_{\circ}}\left(n_{\circ} \leq n\right)$, then, noting that there are 43 -Sylow subgroups of $G / H$, we get $p^{n}-1=4\left(p^{n_{\circ}}-1\right)$, or equivalently, $p^{n_{\circ}}\left(p^{n-n_{\circ}}-4\right)=-3$. This clearly implies $p=3, n_{\circ}=1$, and $n-n_{\circ}=1$. Therefore $|H|=9$. But we know that either $H$ is normal minimal or $H / L$ is isomorphic to $C_{3} \times C_{3}$. Hence, $H$ is minimal normal and isomorphic to $C_{3} \times C_{3}$. It is now easy to see by the Frattini argument, and noting that $H$ is minimal normal, that $G$ is isomorphic to a semidirect product of $\operatorname{SL}(2,3)$ and $C_{3} \times C_{3}$. This proves Step 4 and the lemma.

Proof of Theorem 3.1. By Lemma 3.2, we only need to consider the case that ker $\chi=K>1$. So suppose $K>1$ and that the theorem is false. Let $G$ be a minimal counterexample. If $K$ is not minimal normal in $G$, let $N<K$ with $N \triangleleft G$. Then considering $\chi$ as an irreducible character of $G / N$, we see that $G / N$ satisfies the hypotheses of the theorem with ker $\chi=K / N>1$, and so by the minimality of $G, G / N$ is isomorphic to one of the groups listed in (c), (d), and (e) of the conclusion of Theorem 3.1. This implies $\chi(1)=2$ or 3 . But then, Lemma 3.6 and 3.7 imply that $G$ itself is isomorphic to one of the groups listed in (c), (d), and (e), contrary to our assumption that $G$ is a counter-example. Therefore we may assume $K$ is minimal normal in $G$. By Lemma 3.4, $G / K$ is a Frobenius group of the type listed in (b) and if $M / K$ is the Frobenius kernel, then $M^{\prime}=K$. We can now apply Lemma 2.16 (see Remark 3.3(iii)) and conclude that one of the following three holds.

(i) $|M / K|=3$,

(ii) $M$ is a $p$-group for some prime $p$, 
(iii) There exists an irreducible character $\varphi \neq 1$ of $K$ and $\theta$ and irreducible character of $G$ such that $\left(\varphi^{G}, \theta\right)_{G} \geq 1$ and $(\theta(1), q-1) \neq 1$.

If $|M / K|=3$, then $\chi(1)=|M / K|-1=2$ and so by Lemma 3.6, $G$ is isomorphic to $S_{4}$, contrary to the assumption that $G$ is a counterexample. If $M$ is a $p$-group, then by Lemma $3.5, G$ is isomorphic to $\mathrm{SL}(2,3)$, again contradicting our assumption. So statement (3) above holds. Since $\chi(1)=q-1$, $\chi=\theta$ by hypotheses. Then $\left(\varphi^{G}, \chi\right)_{G}=\left(\varphi,\left.\chi\right|_{K}\right)_{K} \geq 1$. But $\left.\chi\right|_{K}=\chi(1) \cdot 1_{K}$ whereas $\varphi \neq 1_{K}$. This is once again a contradiction and the proof of Theorem 3.1 is complete.

Remark 3.8. We observe that there is no group satisfying the hypotheses of the Theorem 3.1 with $\operatorname{ker} \chi=K>1$ and with $(\chi(1), 6)=1$.

\section{Acknowledgments}

The author is grateful to Professor Gary M. Seitz for his many helpful suggestions during the preparation of this paper.

\section{References}

[1] C.W. Curtis, The Steinberg character of a finite group with a $(B, N)$-pair, J. Alg. 4 (1966), 433-441.

[2] C.W. Curtis and I. Reiner, Representation theory of finite groups and associative algebras, Interscience, New York, 1962.

[3] S.W. Dagger, On the blocks of the Chevalley groups, J. Lon. Math. Soc. (2) 3 (1971), 21-29.

[4] W. Feit, Groups with a cyclic Sylow subgroup, Nagoya Math. J. 27 (1966), $571-584$.

[5] W. Feit, Characters of finite groups, Benjamin, New York, 1967.

[6] P.X. Gallagher, Group characters and normal Hall subgroups, Nagoya Math. J. 21 (1962), 223-230.

[7] D. Gorenstein, Finite groups, Harper and Row, New York, 1968.

[8] M. Hall, Jr., The theory of groups, MacMillan, New York, 1959. 
[9] B. Hupperrrt, Endliche Gruppen I, Springer-Verlag, Berlin, 1967.

[10] I.M. Isaacs, Fixed points and characters in groups with non-coprime operator groups, Can. J. Math. 20 (1968), 1315-1320.

[11] G.M. Seitz, Finite groups having only one irreducible representation of degree greater than one, Proc. Amer. Math. Soc. 19 (1968), 459-461.

[12] G.M. Seitz, M-groups and the supersolvable residual, Math. Zeitschr. 110 (1969), 101-122.

[13] J.G. Thompson, Normal p-complements and irreducible characters, J. Alg. 14 (1970), 129-134. 